dependence), cigarette and EC use, and nicotine craving and withdrawal at baseline and week 6 . Cotinine and exhaled carbon monoxide were assessed at baseline and week 6. RESULTS/ANTICIPATED RESULTS: Participants who completely switched from smoking to ECs (exclusive EC users) and those that partially switched (dual users), maintained cotinine levels ( $p s>.05$ ) and showed reductions in cigarette dependence and withdrawal (ps<.01). However, exclusive EC users showed no significant changes in total nicotine dependence from baseline to week $6(\mathrm{p}=.123)$, while dual users showed increased total nicotine dependence $(\mathrm{p}<.001)$. Dual users displayed similar levels of EC dependence as exclusive EC users but a lesser reduction in cigarette dependence. Exclusive EC users and dual users showed reductions in craving and withdrawal from baseline to week 6. DISCUSSION/SIGNIFICANCE OF FINDINGS: This study is among the first to prospectively examine changes in dependence, craving, and withdrawal among an understudied sample of smokers making a switch attempt. Smokers who completely switch to ECs maintain nicotine levels and dependence, suggesting that they have a similar reinforcement profile to cigarettes and facilitate switching.

57963

The Impact of Asian American Perceived Discrimination on Health Utilization

Thomas Le, BS ${ }^{1}$, Gilbert Gee, PhD² , Lorraine Dean, ScD ${ }^{1}$, Hee-Soon Juon, MSN, PhD ${ }^{3}$ and Som Saha, MD, MPH ${ }^{1}$

${ }^{1}$ Johns Hopkins Bloomberg School of Public Health, ${ }^{2}$ UCLA Fielding School of Public Health and ${ }^{3}$ Thomas Jefferson University

ABSTRACT IMPACT: Understanding how perceived discrimination affects Asian Americans can help stakeholders target subgroups that are at highest risk of discrimination-related behaviors and design culturally appropriate interventions to ensure equitable access to healthcare. OBJECTIVES/GOALS: The COVID-19 pandemic has exposed longstanding anti-Asian racism in the US. Yet, effects of discrimination on Asian American health are unknown, partly because diverse Asian American populations are analyzed in aggregate. We aim to understand how perceived discrimination affects healthcare utilization among different Asian American subgroups. METHODS/STUDY POPULATION: We examine the association of perceived discrimination with healthcare utilization using the California Health Interview Survey (CHIS). In the CHIS, respondents reported whether they would've gotten better medical care if they belonged to a different race. We examine the association between these responses and physician visits within the past year, in the survey years 2003, 2004 and 2016-2017. We adjust for covariates based on the Andersen Health Behavior model. Subsequent modeling examines potential mediating and moderating factors such as limited English proficiency, immigration status, income, and survey year. Asian American subgroups analyzed include Asian Indian, Korean, Chinese, Filipino, Vietnamese, Japanese, and other Asian. RESULTS/ANTICIPATED RESULTS: Results will highlight how perceived discrimination incentivizes or disincentivizes certain Asian subgroups to utilize healthcare. Asian American subgroups have differing and diverse experiences with discrimination due to their historical and cultural differences; results will elucidate how discrimination affects these subgroups. Results will be compared to non-Hispanic Whites, who represent the racial group least likely to experience discrimination in the US. Mediation and moderation analysis will help understand how traditionally cited factors for healthcare utilization interact with perceived discrimination on Asian Americans. DISCUSSION/SIGNIFICANCE OF FINDINGS:
Asian American subgroups are understudied, despite Asian Americans being one of the fastest growing racial groups in the US. Understanding how perceived discrimination affects Asian Americans can help stakeholders target subgroups that are at highest risk of discrimination-related behaviors and design culturally appropriate interventions.

82032

\section{Lessons learned from a virtual engagement salon amidst} a pandemic

Pierre Khalil, Jordan Louis, Barbara Tafuto and Marsha Gordon Rutgers University

ABSTRACT IMPACT: This work is intended to improve community engagement salons both virtually and generally in order to maximize the benefit of this vital research tool. OBJECTIVES/GOALS: The Meharry-Vanderbilt Community Engaged Research Core (CERC) provides a protocol to maintain high standards in community engagement studios, even in a virtual setting amidst a pandemic. A virtual community engagement salon was selected as a case study to evaluate outcomes. METHODS/STUDY POPULATION: A virtual salon regarding sun safety in the Latinx community was observed live via Zoom and as a recording afterward. Following dissemination and completion of the post-meeting surveys, authors compiled and reviewed the results. An assessment was developed to determine the salon's alignment with the Meharry-Vanderbilt CERC guidelines in a virtual setting; this was designated as the primary outcome. Data from the session were compared to the available literature on the topic, which produced three subheadings to the secondary outcome essential to the success of virtual community engagement salons: researcher preparedness, participant selection, and survey importance. RESULTS/ANTICIPATED RESULTS: The CERC guidelines of the community engagement salon were met and were effectively translated into a virtual setting. The presentation given by the researcher followed all technical instructions, yet it was clear that the researcher's demeanor and conversational softskills were lacking. Instead of the recommended bi-directional flow of conversation, the conversation flow shifted to a unidirectional state controlled by the participants. Following the session, only three participants completed the survey along with the researcher. This completion rate of under $50 \%$ provides limited feedback on participants' perspectives on the session's quality and points to improve future sessions. DISCUSSION/SIGNIFICANCE OF FINDINGS: Pre-meeting researcher preparation is necessary to engage community stakeholders effectively. The lack of completed surveys from participants suggests potential fatigue from leading a majority of the conversation. Results demonstrate that solely meeting the requirements of the CERC does not suffice.

92741

\section{Racial differences in patient-reported distress among} women with endometrial cancer ${ }^{\dagger}$

Hadley Reid, Mary Katherine Montes de Oca, Oluwadamilola M. Fayanju, Laura J. Havrilesky and Brittany A. Davidson

Duke University Medical Center

ABSTRACT IMPACT: This work will inform and improve the way we assess and treat distress in women with endometrial cancer. OBJECTIVES/GOALS: Distress from cancer is associated with worse processes of care. Differences in outcomes by race/ethnicity in 